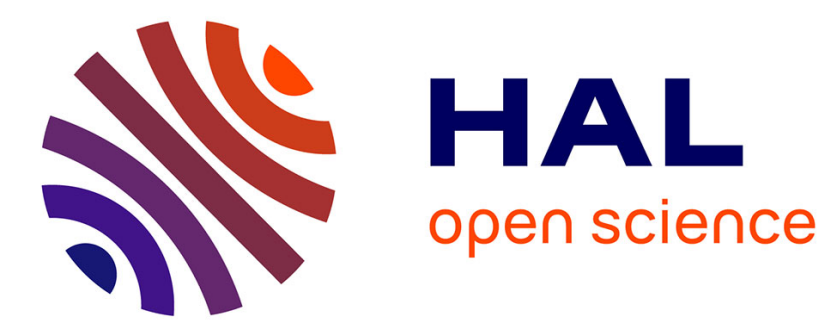

\title{
Confinement and optical properties of the plasmonic inverse-rib waveguide
}

Henri Benisty, Mondher Besbes

\section{To cite this version:}

Henri Benisty, Mondher Besbes. Confinement and optical properties of the plasmonic inverse-rib waveguide. Journal of the Optical Society of America B, 2012, 29 (4), pp.818-826. hal-00683901

\section{HAL Id: hal-00683901 \\ https://hal-iogs.archives-ouvertes.fr/hal-00683901}

Submitted on 19 Feb 2013

HAL is a multi-disciplinary open access archive for the deposit and dissemination of scientific research documents, whether they are published or not. The documents may come from teaching and research institutions in France or abroad, or from public or private research centers.
L'archive ouverte pluridisciplinaire HAL, est destinée au dépôt et à la diffusion de documents scientifiques de niveau recherche, publiés ou non, émanant des établissements d'enseignement et de recherche français ou étrangers, des laboratoires publics ou privés. 


\title{
Confinement and optical properties of the plasmonic inverse-rib waveguide
}

\author{
H. Benisty* and M. Besbes \\ Laboratoire Charles Fabry, Institut d'Optique, CNRS, Université Paris Sud, 2 Avenue Augustin Fresnel, \\ 91127 Palaiseau Cedex, France \\ *Corresponding author: henri.benisty@institutoptique.fr
}

Received November 30, 2011; accepted January 2, 2012;

posted January 17, 2012 (Doc. ID 159065); published March 30, 2012

Plasmonic inverse-rib optical waveguides, consisting of a high-index inverse rib embedded in low-index medium above a flat metallic surface, are investigated under four aspects: (i) the optimal angle $\theta$ of the rib sidewall for tight modal confinement is assessed, (ii) the effect of the geometric parameters and the wavelength on propagation losses is given, (iii) we use a 3D simulation to assess how well light from an emitting dipole is captured by such a tightly guiding structure, and (iv) we show that for two such parallel hybrid waveguiding systems, when one of them has added gain, we have a plasmonic version of the PT-symmetric waveguide arrangement, and we additionally show that complex gain is needed to restore a truly exceptional point in its propagation constant evolution. (c) 2012 Optical Society of America

OCIS codes: $\quad 230.7380,240.6680,350.4238,130.3120,130.4110,250.3680$

\section{INTRODUCTION}

Plasmonic waveguides capitalize on the superior confinement properties of metal confinement to attain very small effective areas. Such miniaturization is interesting either for implementing photonic functions at the nanoscale (e.g., in nanobiophotonics) or for improving fundamental light-matter interaction processes such as spontaneous emission ( $\mathrm{SpE}$ ). Plasmonic lasers, so-called "spasers," emerged in 2009 [1,2]. In one preferred form, they exploit the special guidance that arises between a metallic plane and a high-index channel, separated by a thin lower index gap [3]. This is a class of hybrid plasmonic waveguiding, with assistance from highindex dielectric confinement and thus mitigation of the loss/confinement compromise [3- -5$]$.

We have recently proposed, inspired by this spaser achievement, a hybrid geometry shown in Fig. 1(a) that we call the plasmonic inverse-rib optical waveguide (PIROW) [5]. An important aspect of our PIROW proposal is that the metal can remain unpatterned, while the fabrication technology can be made very simple, direct, and versatile through exposure and spin-coating of known available resists, say polymethyl methacrylate. In a slightly simplified view, a low-index (L) resist is coated, exposed, and developed so as to form a trench, but not traversing down to the metal. Then a high-index resist (H) can nearly planarize the structure and fill the trench. A tightly confined plasmonic mode then appears at the tip of the trench cross section, typically with TM-like polarization. It is rapidly evanescent in both $x y$ directions of the plane. The metal/H/L sequence helps confining the large field region to the $\mathrm{L}$ gap below the inverse ridge. This configuration increases crucial figures of merits such as the Purcell factor, and is certainly advantageous to elaborate devices with subwavelength resolution. Our proposed implementation also makes it easy to modulate the guide parameters by structuring only the low-index medium. It can easily be exploited to take advantage of the rich background of distributed Bragg reflector (DBR) structures or of 2D photonic crystals.

Currently, hybrid plasmonic waveguide proposals are emerging, using a low-index dielectric between a metal and a waveguiding higher index dielectric [6-9]. They often assume "square" geometries that are derived from simplified processing considerations, such as a preference for vertical etching and silicon-oriented applications. Our purpose, exploiting simpler technology and targeting near-infrared wavelengths for biophotonics, is complementary. So, clearly, a few clever aspects outlined in these proposals could be combined with ours. We believe that the smooth interfaces of the PIROW remain a crucial asset.

We first complement our first paper [5] in terms of the passive PIROW properties by analyzing the role of the inverse-rib sidewall angle and also by studying the trends on losses in more detail. But "active" properties of plasmonic-based systems can also be of high interest, and two such properties are discussed in this paper as follows.

Channeling or concentrating a point emitter's light in a single mode, the so-called $\beta \mathrm{SpE}$ factor, is relevant for applications to sensing, e.g., capturing light with subwavelength resolution from fluorescent probes attached to analytes. It is well documented that this $\beta$ factor is controlled by the environment-dependent radiative lifetime, the well-known Purcell factor. We therefore calculate the $\beta$ factor and the Purcell factor at key locations, and we test how much metal proximity quenches radiative emission.

We also investigate coupled waveguide devices with balanced gain and losses, a more exploratory issue. Photonic systems with balanced gain and losses may show special properties related to their parity-time symmetry (PT symmetry), which may or may not be broken by their field eigenmodes [10]. Symmetry breaking is of high practical interest because it causes exceptional points in the gain dependence of the eigenvalues, with singular trends around them that may inspire 
novel optical designs, notably those related to "unidirectional" behavior [11-21]. We recently outlined [22] the possibility to get the essential features of perfect PT systems in this respect in cases where losses are fixed, as occurs with metals, and gain variable. In such a situation, the gain/loss balance desirable for the special properties of PT-symmetric systems (such as an exceptional point in the propagation constants with singular behavior around it), holds only around a single gain value with some margin around it. We will discuss how our general statements of [22] apply to a pair of PIROW with gain added in one of them, and we will notably indicate a way to restore a better exceptional point than the one found when applying only the prescriptions of [22]. By "better," here, we mean that the smoothing of the singularity as caused by the imperfect PT symmetry is healed by feasible parametric modifications of the active (gain) waveguide, namely by the use of complex gain with simultaneous variations of real and imaginary parts of the index.

The procedure is as follows: first, with a two-dimensional (2D) solver, we examine the optimal angle $\theta$ of the trench sidewall, the "optimal wedge," providing maximal confinement. Next, we study how losses behave versus the various parameters of interest: wavelength, angle, and material (here $\mathrm{Au}$ or $\mathrm{Ag}$, to work in the visible or near-infrared). The next section addresses the issue of how well light is captured and guided when it is emitted near, or in, such a PIROW using a three-dimensional (3D) finite element simulation, giving answers such as the Purcell effect or a "SpE factor" ( $\beta$ factor) in radiative modes. Finally, we present the variations of propagation constants for the case of a guide with gain next to a passive one, following the prescriptions of [22]. The smoothing of the exceptional point that occurs is seen to be much larger than in less confined waveguides [22,23], but a way to restore it is demonstrated, which opens new perspectives for the practical use of PT symmetry-breaking effects in plasmonic systems.

\section{ANGLE-DEPENDENT CONFINEMENT}

Here, we vary the angle $\theta$ of the PIROW rib sidewall. The angle and all notations are defined in Fig. 1(b). The other parameters are the same as in our first study [5], namely $n_{L}=$ 1.5 and $n_{H}=2.0$. A gold surface at $633 \mathrm{~nm}$ is chosen, with the indices of Johnson and Christy [24]. Other indices are fixed because they have much weaker spectral variations (polymer, solgel). We use the same $2 \mathrm{D}$ vectorial solver developed in-house, as in [5,25], deployed under a MATLAB environment. The thickness of the $L$ index is $90 \mathrm{~nm}$, and the top high-index layer is $40 \mathrm{~nm}$ thick outside the rib. The rib width $L_{\text {tip }}$ is taken as $20 \mathrm{~nm}$ (as it was in [5], but with $\theta=15^{\circ}$ ) wide. The rib height is $80 \mathrm{~nm}$ so as to leave a $\mathrm{d}=10 \mathrm{~nm}$ low-index gap. In this regime, we have local fields in the tip area mainly determined by electrostatic response to the metal charges with only minimal retardation effects.

Figure 2(a) shows the series of mode profiles for the vertical $E_{y}$ component, cut through the symmetry axis $E_{y}(0, y)$ and through the midgap plane $x z$ parallel to the metal, $E_{y}(x, d / 2)$. In all cases of Fig. $2, L_{\text {tip }}=20 \mathrm{~nm}$. A finer scan was used in Fig. 2(c), giving the effective index variation for this mode, $n_{\text {eff }}=n_{\text {eff }}(\theta)$. As described earlier [5], the evanescence and the tight localization are granted by the effective index inequality $n_{L}<n_{\text {eff }}<n_{H}=2.0$ : the first grants the plasmonic-type behavior, and the second reasonably ensures that the mode is hybrid and is not a mere perturbation of a plasmonic mode at a $\mathrm{Au} / n_{H}$ interface by a marginal effect of the low index, a case in which high effective indices of plasmonic modes $n_{\text {eff }}>n_{H}=2.0$ would arise.

The profile along $y$ thus retains the predominance of the low-index peak field region, even for the limit case $\theta=0^{\circ}$ (vertical walls), which provides only weak high-index confinement. The profiles in the lateral $x$ direction, Fig. 2(b), make it clear what is the key compromise for strong confinement. The field is maximally pinched around its central peak for the lowest $\theta$ values (say $0^{\circ}-10^{\circ}$ ), but the tails are still large. For larger angles, the tails decrease, while the central peak at high field values remains narrow and only broadens for a sidewall angle $\theta>\sim 70^{\circ}$.

An important measure of the degree of confinement is the effective area of the mode. At the present stage, an exact calculation is not critical, and for more clarity [26], we simply determine the extent $\Delta x_{\alpha}$ and $\Delta y_{\alpha}$ of the regions of each chosen axis $\left[E_{y}(0, y)\right.$ or $\left.E_{y}(x, d / 2)\right]$ for which the inequality $\left|E_{y}\right|>\alpha E_{y}^{\max }$ is satisfied with a parametric $\alpha$ ratio. We note that the field $E_{y}(0, y)$ is nearly constant and does not decay much below $E_{y}^{\max }$ across the low-index gap. We simply define the mode area as $S_{x y}(\theta, d, \alpha)=\Delta x_{\alpha} \Delta y_{\alpha}$. If the ratio $\alpha$ is not too small, $\Delta y_{\alpha}$ remains essentially the gap width itself, $\Delta y_{\alpha}=d$. Hence the $x$ confinement is the main characteristic Only for relatively low $\alpha$ does $\Delta y_{\alpha}$ extend above $d$, in which case our definition is still of great relevance, but a more accurate study could be welcome for details.

These effective area data are gathered in Fig. 2(d). The five curves correspond to the increasing ratio $\alpha$, namely, $\alpha=0.45$, $0.5,0.6,0.7$, and 0.8 . The three bottom curves (solid lines) correspond to the regime where $\Delta y_{\alpha}=d$. The two top curves
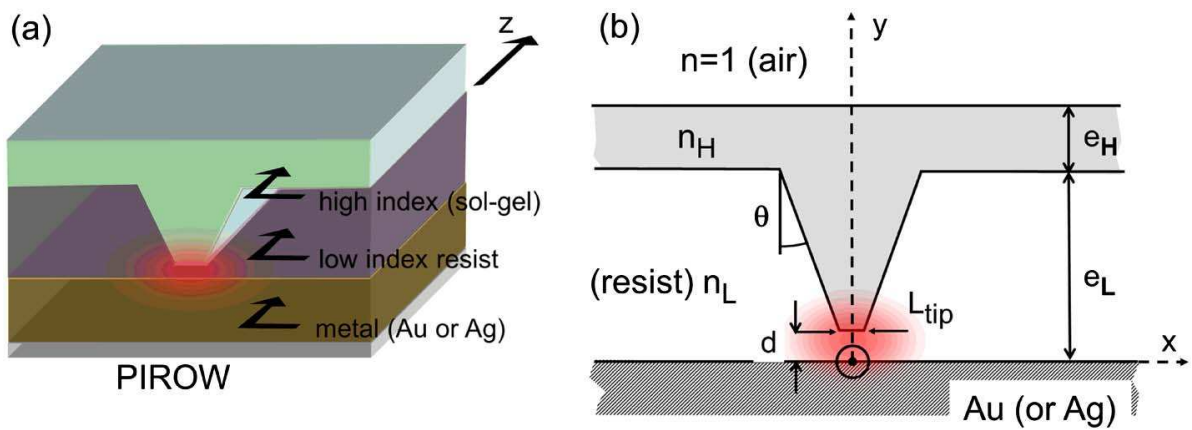

Fig. 1. (Color online) (a) Structure of the PIROW, the red spot being indicative for the confined mode profile and (b) optogeometric parameters of the rib and layer sequence. 

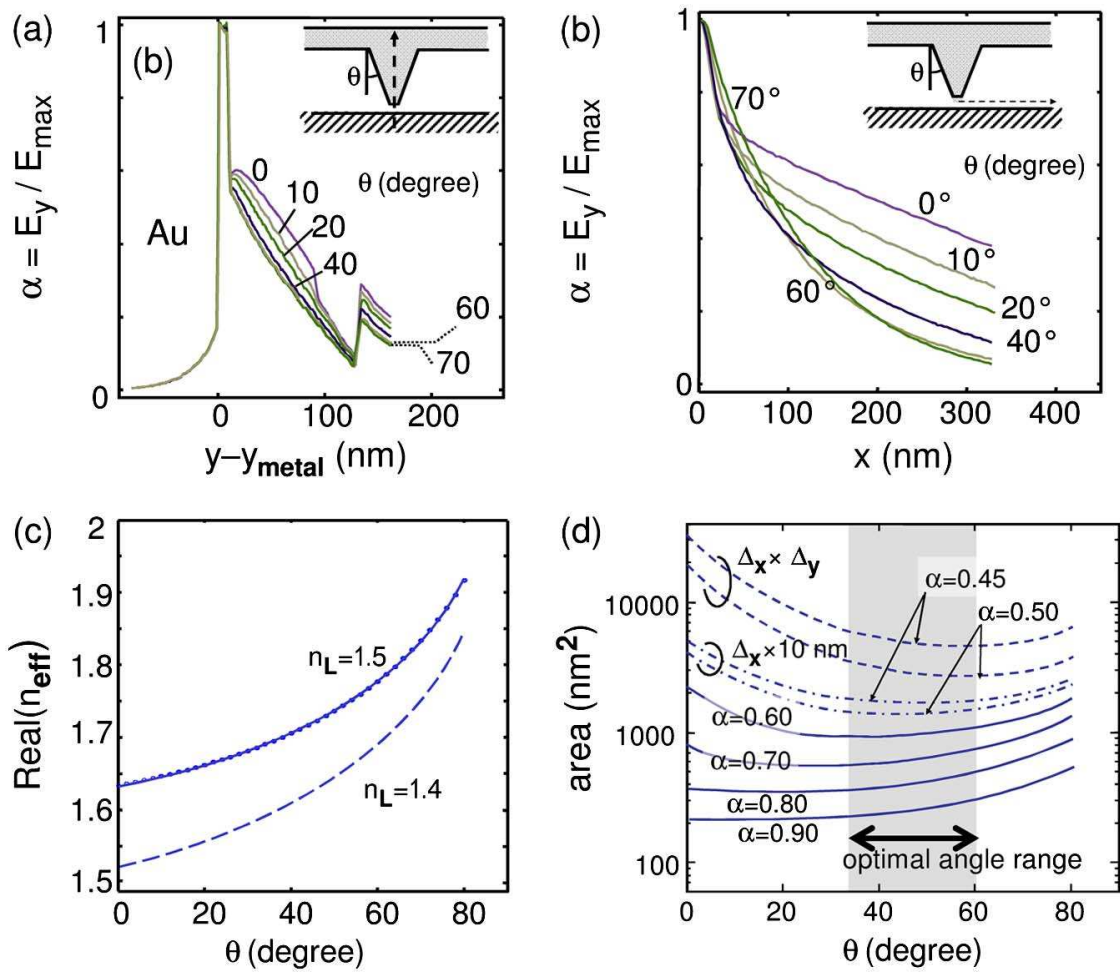

Fig. 2. (Color online) PIROW modal characteristics for tip width $L_{\text {tip }}=20 \mathrm{~nm}, n_{L}=1.40$, and variable inverse-rib angle $\theta$. (a), (b) Field profiles along the cuts indicated in their inset. (c) $\left(\operatorname{Re}\left(n_{\text {eff }}\right)\right)$ trend versus angle $\theta$ (dashed line), with comparison to $n_{L}=1.50$ (solid line with dots). (d) Area enclosed in the range $\left|E_{y}\right|>\alpha E_{y}^{\max }$ versus angle for different values of $\alpha$.

(dashed lines) correspond to the regime where $\Delta y_{\alpha}>d$. The two corresponding products $\left(\Delta x_{\alpha} \times d\right)$ are plotted as a dashed-dotted line to allow for comparison. The main striking point here is that for a not-too-high-ratio, say $\alpha=0.60$, the effective area is optimally confined typically for $\theta$ around $40^{\circ}$. The minimal effective area for the dashed curves (lower $\alpha$ ratio) occurs at a larger angle: first, for this $\alpha$ regime, one looks at the tails of the profile, for which a high effective index is favorable, which comes together with a larger angle $\theta$. And second, for the dashed curves, the decay in the high-index medium plays a role and adds a similar trend [more decay for a larger effective index and a higher $\theta$, Fig. 2(a)].

The typical effective areas for $S_{x y}(\theta, d, \alpha)$ for ratio $\alpha=0.6$ or 0.7 are 5 to $1010^{-4} \mu \mathrm{m}^{2}$, which is the equivalent of $30 \mathrm{~nm} \times$ $30 \mathrm{~nm}$ or less.

We checked the influence of the low-index medium, performing a similar study for $n_{L}=1.50$ instead of 1.40. The effective index curve is indicated by a dashed line in Fig. 2(c). The simple trend, with an offset of $\Delta n \sim 0.1$ on the low-index/low-angle side suggests that, as far as design is concerned, extrapolating an effective index from such limited data can be effective to orient modeling and material choice in a substantial range.

In Fig. 3, we compare the confinement curves for these two cases by plotting side by side the plot of Fig. 2(d) and an equivalent one for $n_{L}=1.40$. There are several trends. Focusing first on the $\Delta x_{\alpha=0.7}(\theta)$ for both indices (associated to halved $|E|^{2}$ ), we see a small advantage for the lower index, with an area $450 \mathrm{~nm}^{2}$ instead of $550 \mathrm{~nm}^{2}$. The advantage is more obvious for smaller $\alpha$, i.e., if we look further into the mode tail. The advantage for $\Delta x_{\alpha=0.45}(\theta)$ in terms of area $\Delta x_{\alpha} \Delta y_{\alpha}$ (dashed lines in the graphs) can be almost a factor of $2,2500 \mathrm{~nm}^{2}$, instead of about $4600 \mathrm{~nm}^{2}$. Note also that the optimal confinement angle generally shifts to larger angles for the smaller $\alpha$ values. This is because the mode index is higher. It implies a faster lateral decay in the low-index material, which is, however, appearing clearly only if we look far enough in the tails and not too close to the tip, as the large angles
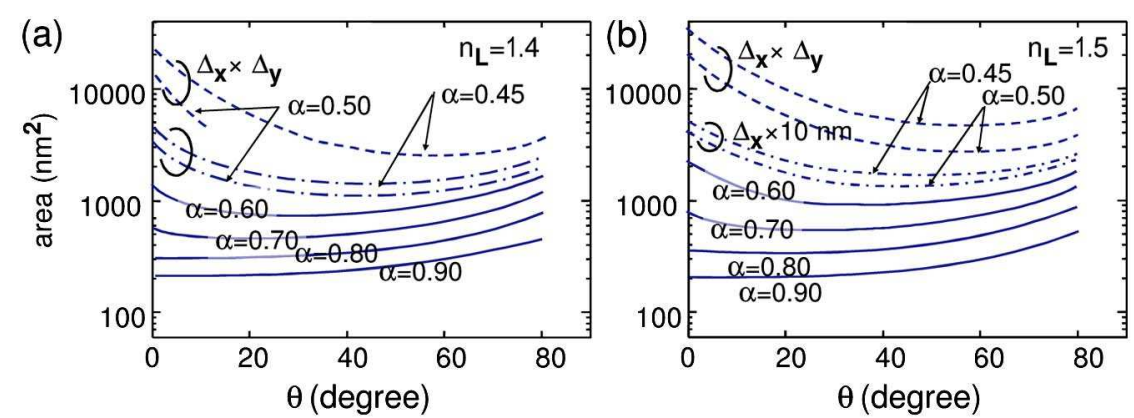

Fig. 3. (Color online) Comparison of confinement for two low-index values differing by $\Delta n=0.1$, as a function of the inverse-rib sidewall angle: (a) $n_{L}=1.40$ and (b) $n_{L}=1.50$. 
tend to limit the field profile downward curvature around the tip (for a given gap thickness).

The overall trends are an indication that it is essentially worth making use of either a lower index first layer, or similarly, a higher index top layer, but this interest is modulated by the details of the figure of merit.

\section{LOSSES OF A PLASMONIC INVERSE-RIB WAVEGUIDE}

In spite of the unavoidable losses of passive metallic structures, there are several situations where short propagation lengths are of interest, because of their unique tighter confinement. The strategy is to next channel the information to a larger, less dissipative channel waveguide.

The $633 \mathrm{~nm} \mathrm{He-Ne} \mathrm{laser} \mathrm{wavelength} \mathrm{is} \mathrm{convenient} \mathrm{in} \mathrm{sev-}$ eral experiments. We hence start by looking in Fig. 4 at the imaginary effective index as a function of PIROW angle for this case and for the index $n_{L}$ equal to 1.40 . The trend is quite smooth, increasing gently at larger angles. The large angle limits the tip effect and, thus, forces the field to interact with the metal on a broader $x$ range. Note that the trend is similar to the trend of $\Delta x_{\alpha=0.9}(\theta)$, suggesting that the key factor to minimize losses is to get the tightest lateral $(x)$ confinement, whatever length the field tail has along $y$-Fig. 2(b). At the typical $45^{\circ}$ angle where confinement is optimal under broad criteria, the losses are 10\%-15\% above their minimum value (obtained at vanishing $\theta$ ).

Next, we look at the spectral dependence of the losses. In Fig. 5, we plot the imaginary part of the effective index versus wavelength $\lambda$. The result helps us to check that the trends of the weak positive angular dependence for $633 \mathrm{~nm}$, while not being completely general, are fairly well reproduced at all wavelengths in the range from yellow $(570 \mathrm{~nm})$ to nearinfrared $(870 \mathrm{~nm})$. The imaginary index rise on the short wavelength side is due to the Au green absorption band.

The limit value on the long wavelength side is about $\operatorname{Im}\left(n_{\text {eff }}\right)=0.01$. This is not large in absolute terms. But it would nevertheless require a strong material gain in one of the dielectric media to get net gain, typically thousands of $\mathrm{cm}^{-1}$, depending on the exact overlap factor. This defines in which limit our PIROW could be used for a spaser with $\mathrm{Au}$. Such gain-carrying waveguides are addressed in the next section on PT symmetry breaking.

Gold is the preferred metal for technological realizations, being robust against aging and oxidation. Silver works at shorter wavelengths, and it also has slightly less losses.

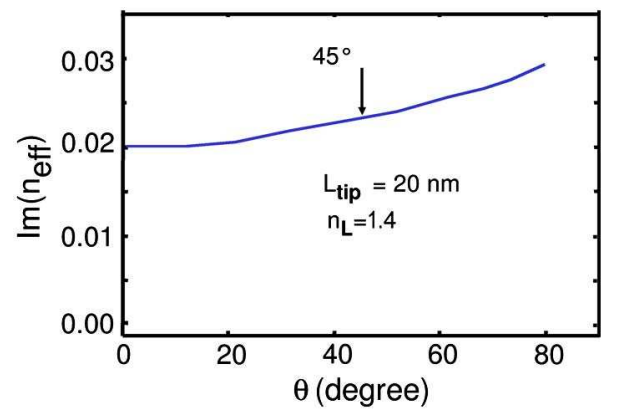

Fig. 4. (Color online) Losses as a function of the inverse-rib angle at $633 \mathrm{~nm}$, given through the imaginary effective index. Note however the substantial real effective index variation on the same range in Fig. 2 .

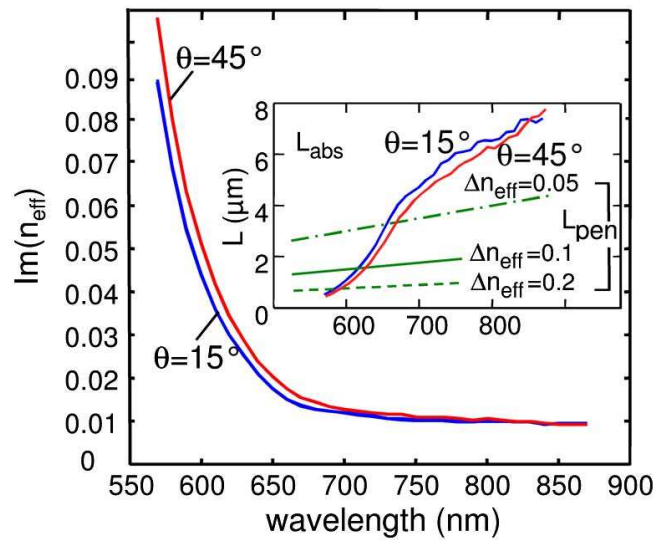

Fig. 5. (Color online) Losses as a function of wavelength for a $45^{\circ}$ PIROW and $15^{\circ}$ PIROW as indicated, using gold. Inset, the associated absorption lengths that become larger than a typical penetration length in a periodic system of given modulation $\Delta n_{\mathrm{eff}}$ at wavelengths between 600 and $700 \mathrm{~nm}$.

The comparison of Ag-PIROW and Au-PIROW imaginary modal effective indices in Fig. 6(b) shows a gain of about 10\%-20\% in the near-infrared, while in the range $550-600 \mathrm{~nm}$, the $\mathrm{Ag}$ advantage is obvious and large (factors of $>5$ ). We note in Fig. 6(a) that Ag characteristics also result in a weaker real index.

In both Figs. 5 and 6 , the inset translates the imaginary index into an absorption length and compares the result to a penetration length of a Bragg mirror, calculated with the naive formula for the indicated index contrast (as done in [5]) $\Delta n_{\text {eff }}$. A shallower angle brings some more margin for

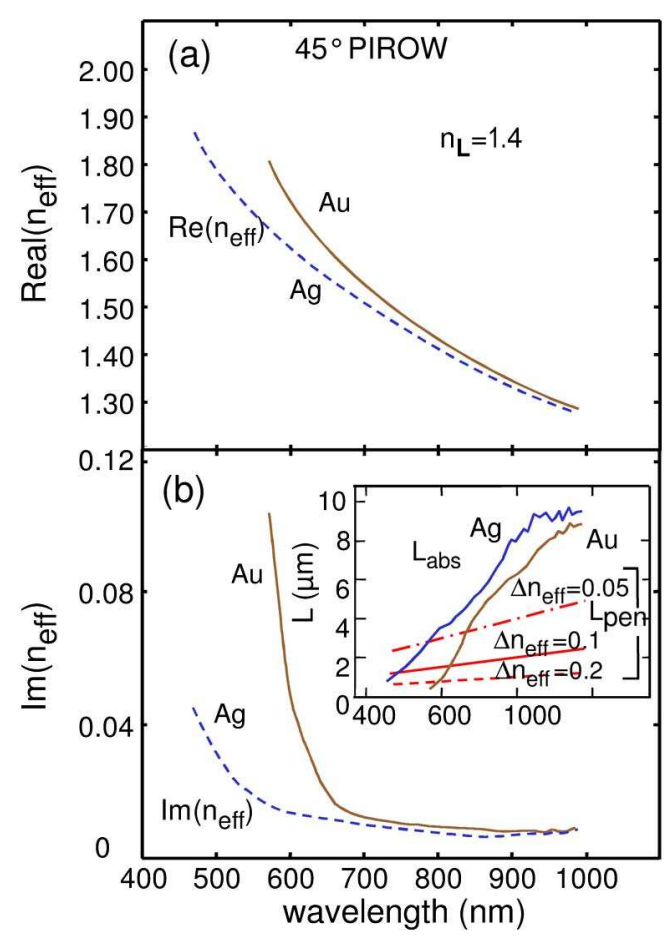

Fig. 6. (Color online) (a) Effective index versus wavelength for a $45^{\circ}$ PIROW with Au (top curve) or Ag (lower curve) and (b) same as Fig. 5, but comparing Ag (dashed line) and Au (solid line) losses $\operatorname{Im}\left(n_{\text {eff }}\right)$. The inset transforms the same comparison into an absorption length one, as in Fig. 5. Note that Ag may behave well at wavelengths as short as $550-600 \mathrm{~nm}$. 
$\mathrm{Au}$ (Fig. 5) to get Bragg reflection at $633 \mathrm{~nm}$ for instance. But Ag gives the largest margin, allowing modest contrasts to provide Bragg reflections down to roughly $\lambda=550 \mathrm{~nm}$.

We wish in the near future to calculate what degree of reflection is provided by various shape modulations and how radiation losses due to scattering at transitions can be minimized, a familiar issue of the resonant waveguide grating studies (see, for instance, the collective COST 268 study [27]). Alternatives are to vary the gap width, tip width, or tip angle, or a combination of these. Fabrication constraints do not allow a 3D management of the shape, so the dose variations in $e$-beam lithography should result in a combination of the variation of the three parameters.

\section{LIGHT CONCENTRATION CAPABILITIES}

The enhanced confinement of hybrid plasmonic waveguides translates into attracting possibilities to channel SpE of emitters situated in them or nearby. The overall enhancement of electromagnetic channels for $\mathrm{SpE}$ can be seen through the Purcell factor, $F_{p}=\tau^{-1} / \tau_{o}^{-1}$, the ratio of $\mathrm{SpE}$ rate in the structure $\tau^{-1}$ given as an inverse lifetime, to that in the bulk material where it actually lies, $\tau_{o}^{-1}$. In our case, we expect the guided PIROW mode to be the main actor to enhance SpE rate. A high Purcell factor represents a favorable factor for good light collection only if it does not correspond to extra dissipation in metal, but to actual collection in a guided mode. The associated measure of the degree of collection, more precisely of its ratio to all radiation channels in a single mode, is the "SpE factor," commonly denoted as the $\beta$ factor.

Here the distinction among channels may look somewhat ambiguous because in the long-range (several micrometers), all the hybrid mode energy ends up being dissipated in the metal. However, for short lengths, about $1 \mu \mathrm{m}$, light channeled in a guided mode starts to be reasonably distinct in space from that emitted elsewhere, while absorption of the mode has still been very modest. We used a $3 \mathrm{D}$ finite-element method, which was developed in-house using a LU solver [25]. It includes an electric dipole source to measure these effects. As for geometry, we investigate the emission for a dipole situated at two sets of positions: the plane at midheight of the low-index dielectric gap and the vertical symmetry plane. In both cases, only a quarter of the real space can be modeled, thanks to the two vertical structure symmetry planes. We only use a vertical dipole, even at $x \neq 0$, but we force solutions to be electrically odd or even at the $x z$ symmetry plane and combine the resulting fields adequately to get the full solution (neither odd nor even in general). We model a system with the following parameters:

The gap height is $d=10 \mathrm{~nm}$, tip width is $L_{\text {tip }}=20 \mathrm{~nm}$, rib height is $80 \mathrm{~nm}$, and the top high-index layer is $40 \mathrm{~nm}$ thick. The high index itself is $n_{H}=2.0$, and the low index is $n_{L}=1.4$ to favor a tighter mode profile fitting in a small simulation box. We study the case of modest loss, which means larger wavelength, but we go no further than $\lambda=700 \mathrm{~nm}$ so that far-field effects are reached as closely as possible for the same reasons of limited simulation box. The domain of study, i.e., four times the computational domain given the symmetries, is $1.0 \mu \mathrm{m} \times$ $1.4 \mu \mathrm{m}$ (along the guide axis) $\times 0.42 \mu \mathrm{m}$ (height), comprising $0.2 \mu \mathrm{m}$ of gold in height. We also make use of an embedded subdomain of collection with reduced size to check that the emitted power is no more in the near-field regime but essentially in the propagation regime at the edge of the large domain. The expected difference between this subdomain and the nominal one is then essentially the weak absorption in the waveguide, only a few percent, whereas nonguided near-field components would still feed the metal's Joule losses. This subdomain is here $0.8 \mu \mathrm{m} \times 1.1 \mu \mathrm{m}$ (along the guide axis) $\times$ $0.42 \mu \mathrm{m}$ (height).

In Fig. 7(a), we show the evolution of the Purcell factor $F_{p}$ for a dipole on the symmetry plane at a variable height $z$, from the metal proximity on through the two indices. We find a large enhancement of $F_{p}$ in the low-index gap on the left of the expected discontinuity, $F_{p} \sim 30$ at least, increasing dramatically in the vicinity of metal, as near-field components are allowed to feed Joule losses. This situation has been well identified and is cousin to the one of slot waveguides. The decrease of $F_{p}$ for a dipole lying upward in the high index is clearly related to the mode profile, the $50 \mathrm{~nm}$ closest to the tip providing $F_{p}>3$ typically. The other curve on this plot relates to the sidewall of the inverse rib, on the inner $(H)$ side. Hence the decrease is globally similar to the one on the axis, but somewhat faster, because we are at a less intense field in the guided mode profile at this locus as compared to the axis. However, an enhancement exists in the lower $\sim 7 \mathrm{~nm}$ close to
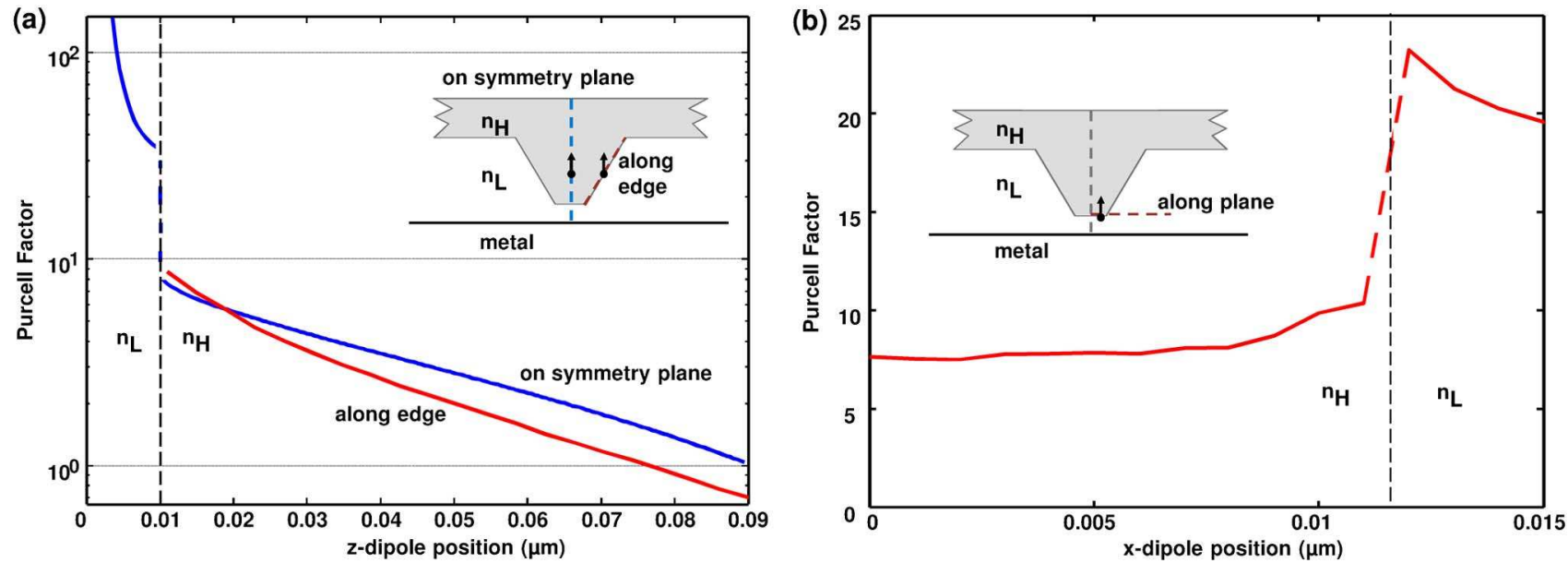

Fig. 7. (Color online) Purcell factor $F_{P}$ for a vertical dipole at wavelength $\lambda=700 \mathrm{~nm}$ in a PIROW: (a) dipole at variable height, either in the symmetry plane $x=0$ or along the rib edge and (b) dipole lying along $x$ at height $z=d$, the height of the tip bottom. Insets, scanned positions. 
the tip over the on-axis position. We attribute it to the corner of the tip, which brings the low-index and its related enhancement to "surround" more the dipole than at the tip center, a point further clarified below.

To further study the situation of emitters at the tip, we show in Fig. 7(b) the Purcell factor along the line at height $z=d$, still for a vertical dipole. In agreement with the above trends, it increases slightly when going to the tip's very corner. Then it increases when going sideways to the low index, but it decays further away from the tip. The evolution can be explained by considering the component of the dipole normal to the $45^{\circ}$ tilted interface, thus a large component for which the situation amounts to a dipole normal to a surface between two dielectrics. In such a case, the Purcell factor counterintuitively peaks on the low-index side and drops on the high-index side, essentially because the dielectric image dipoles are in phase and out of phase respectively. This is documented in detail since, e.g., Lukosz and Kunz's analytical work [28]. Here we have a corner, so the dielectric image effect is not as perfect, but given the $135^{\circ}$ inner angle of the high dielectric, the picture holds. Such effects might also be invoked to explain the low Purcell factor $F_{p}<1$ at the rib's basis, the extreme right of Fig. 7(a).

In Fig. 8, we show the amount of light collected on the sides of the simulation volume (four times the calculation volume thanks to symmetry) as indicated by the inset. The use of the mere surface integration is a rather crude approximation for replacing a calculation of the modal power, but it is also relevant if one implements only a short length of small-gap highly confined PIROW to capture emission from localized emitters, and one further couples or tapers the far end of the PIROW to a purely dielectric waveguide with a larger mode area, thus providing in principle a recapture of most of the emitted light. Our check of the physical significance makes use of a set of two nested collection boxes shown in the inset. We collect more power in the volume of the inner box (length $0.8 \mu \mathrm{m}$ instead of $1.0 \mu \mathrm{m}$ ) than in the larger box because the guided flux in the PIROW decays in the metal when propagating from the small to the large box boundary. But the difference is only a few percent on such a short path

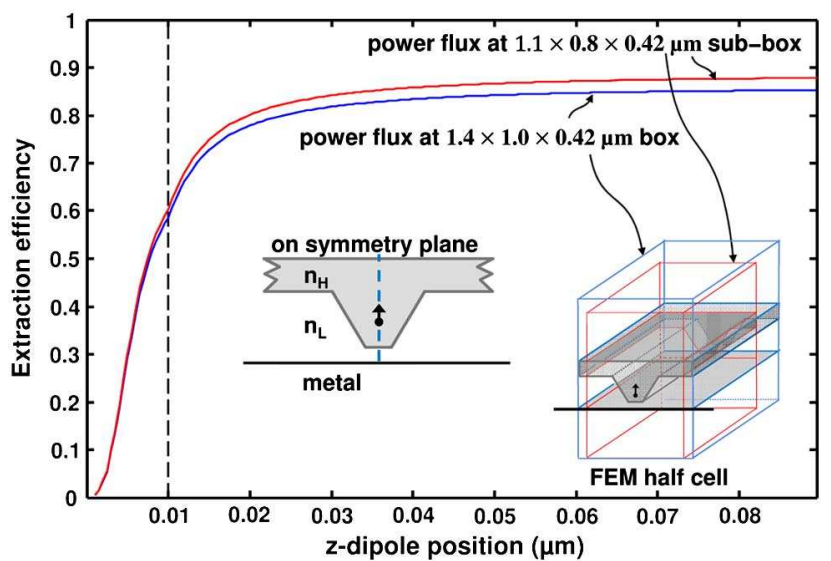

Fig. 8. (Color online) Light capture efficiency by a PIROW on both sides for a dipole lying along the vertical direction at $x=0$. Insets show (left) the scanned position and the nominal and (right) the two "nested" collection boxes used to check that extraction is in the far-field regime. difference, thus, the curve is a good approximation of the degree of capture in the waveguide mode.

For a dipole lying in the low-index gap, close to the metal, this capture is weak because of the metal proximity, typically $30 \%$ on the average. The nearest emitter locations in the lowindex gap tend to feed the metal almost exclusively, thus mitigating the interest of the larger Purcell factor. After a noticeable change of slope upon crossing upward the $\mathrm{L} / \mathrm{H}$ interface, the collected fraction still grows, and a good compromise typically arises $10 \mathrm{~nm}$ above the tip interface, $20 \mathrm{~nm}$ above the metal, where the Purcell factor is still reasonably large (around 6-7). Thus, we confirm in our novel structure the trend, now well-established [29,30], according to which the optimal distance to capture an emitter's light enhanced near a metallic lies in the range $\sim 10-20 \mathrm{~nm}$ for the range of wavelength investigated.

A next step would be to transfer the PIROW modal excitation to a lossless waveguide for instance, with many transition or tapering schemes available [31].

\section{COUPLED GUIDES WITH GAIN AND LOSS: ATTAINMENT OF EXCEPTIONAL POINT}

Fascinating avenues have been recently opened regarding optical systems with PT symmetry and fields which are symmetry-breaking in these structures [11-21]. Systems with balanced gain and losses with a symmetry plane that exchanges them provide a realization of PT symmetry. The eigenvalues of these structures (wave vectors for a frequency in real space) can remain real in the presence of gain, up to an exceptional point where complex eigenvalues appear, with a singular behavior, quadratic instead of linear versus gain around this point. The possibilities explored until recently did not comprise plasmonic structures, or more precisely only "passive PT-symmetric" structures with metal [17], but with no or little gain, thus missing the exceptional point. Plasmonic structures have, in practice, fixed losses. We recently pointed out that by proper adjustment of coupling constant to losses in a pair of coupled guide, one of which has such fixed losses, a singular point with the transition from zero to nonzero imaginary eigenvectors could occur, with a potential for enhanced modulation [22]. We used the so-called LRSPP (long-range surface plasmon polariton) supported by thin gold films and coupled to dielectric waveguides [23] to demonstrate the plasmonics + PT-symmetry-breaking combination. We also introduced coupled PIROW waveguides, but we did not discuss actual simulations with gain in one of them.

We discuss a PIROW-based version of such systems here. We first discuss a basic configuration that might naturally arise if localized photopumping is performed, which is to pump only the inverse rib of the gain-carrying waveguide. More realistically, we also include pumping in the shaded region of Fig. 9(a) above the inverse rib, within circular boundaries centered on the rib apex, as would qualitatively occur with reasonably localized photopumping of the active highindex material.

The parameters are $n_{L}=1.5 n_{H}=2.0$, angle $\theta=40^{\circ}$, wavelength $\lambda=750 \mathrm{~nm}$, to minimize losses in gold and introduce more realistic gain values. The thickness of the $L$ layer is $90 \mathrm{~nm}$ and that of the $H$ layer on it is now $80 \mathrm{~nm}<40 \mathrm{~nm}$ above, to provide more modal gain with a given gain material, 
(a)

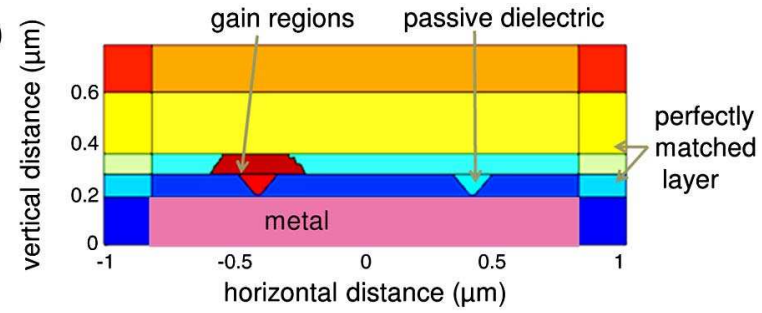

(b)

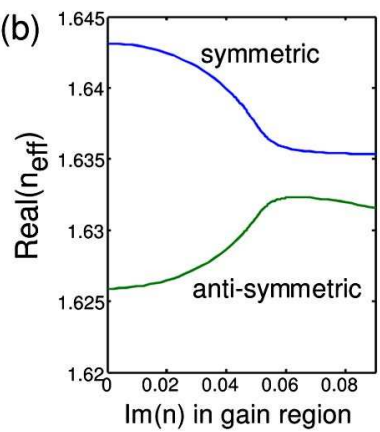

(c)

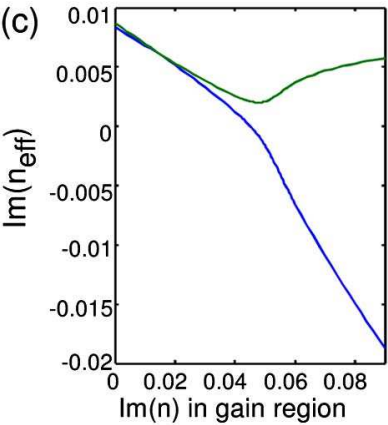

Fig. 9. (Color online) (a) Map of coupled PIROW guides with attempt to implement PT symmetry onto the left one with the same variable added gain $g$ in the inverse ridge and in the shaded region above it, (b) real part of $n_{\text {eff }}$, and (c) imaginary part of $n_{\text {eff }}$

without noticeably affecting the tip effects. The low-index gap left below the rib is $10 \mathrm{~nm}$, so the inverse rib is $80 \mathrm{~nm}$ tall, and the tip width is again $L_{1}=20 \mathrm{~nm}$.

The resulting effective indices $n_{\text {eff }}$ (三eigenvalues) as a function of material gain $\operatorname{Im}(n)$ are shown in Fig. 9. In this case, the coupling distance (center-to-center) was chosen to be $d=$ $0.79 \mu \mathrm{m}$ so as to balance coupling with fixed losses, as discussed in [22]. For a single guide, we first find from modal calculations with gain the coefficient between material gain and modal gain to be $\operatorname{Im}\left(n_{\text {eff }}\right) \approx 0.16 \operatorname{Im}(n)$.

The splitting point where broken-symmetry solutions show up is then located around zero gain $\left[\operatorname{Im}\left(n_{\text {eff }}\right) \sim 0\right]$, but the curves are quite smooth and wash out the expected abrupt singularity. We had shown in [22] such a discrepancy, but much smaller. It becomes here a drawback for the strong modulation of waveguide transmission versus gain, because

the strong modulation is fundamentally based on a high partial derivative of eigenvalues versus modal gain, for instance just above the exceptional point, $d \operatorname{Im}\left(n_{\text {eff }}\right) / d g \gg 1$ in the ideal case. Modal gain decreases to the asymptotic value $1 / 2 \times$ \{isolated guide gain\} at large gains (for the eigenvalue of the symmetric mode).

Our current understanding of this discrepancy with an ideal singularity is that instead of having a real coupling constant as initially assumed in coupled mode theory (CMT) of the coupled guides, we have a complex coupling constant, which becomes more complex as gain grows on the gaincarrying waveguide. More precisely, what counts in solving the CMT model is the product of the two coupling constants $K=\kappa_{a b} \kappa_{b a}$. And it is this product that becomes complex when the system is not a perfect PT-symmetric system, notably due to geometric reasons. Here metallic losses are present on both sides and dielectric gain on one side only, making the situation quite nonideal with respect to PT symmetry.

Prompted by this general idea, we attempted to "heal" the coupling constant to make it real by introducing a compensating region. A change of the real part of the index was added so as to tune the ingredients of $\mathrm{K}=\kappa_{a b} \kappa_{b a}$. Furthermore, because $\mathrm{K}$ becomes complex due to the variable added gain, the index modification has to be proportional to this gain; in other words, a complex index variation has to be introduced instead of gain, with a given complex angle. We chose here to modify only the $n_{H}$ region above the inverse rib, shaded in Fig. 9(a), and not to modify the inverse-rib region, i.e., to leave it with the same index $\operatorname{Re}(n)=\left(n_{H}\right)$ and $\operatorname{Im}\left(n_{H}\right)$ varying as previously, for simplicity. Based on the above discussion, we represent the effect of pumping as an adjustable combination of the imaginary and real part to the complex index, of the form $\left(\tilde{n}_{H}\right)=\left(n_{H}\right)+i g(1+i \alpha), \alpha$ being here a coefficient $<1$. Such a modulation could arise in practice from electro-optical effects if a different dye or a different pumping wavelength is used in this area.

By adjusting the parameter $\alpha$, we could obtain a gradually sharper singularity, and heal it to a large degree in a substantial range: for the value $\alpha=-0.174$, obtained here by trial and error, Fig. 10 shows that the singularity is almost entirely restored. We believe that this methodology for healing the
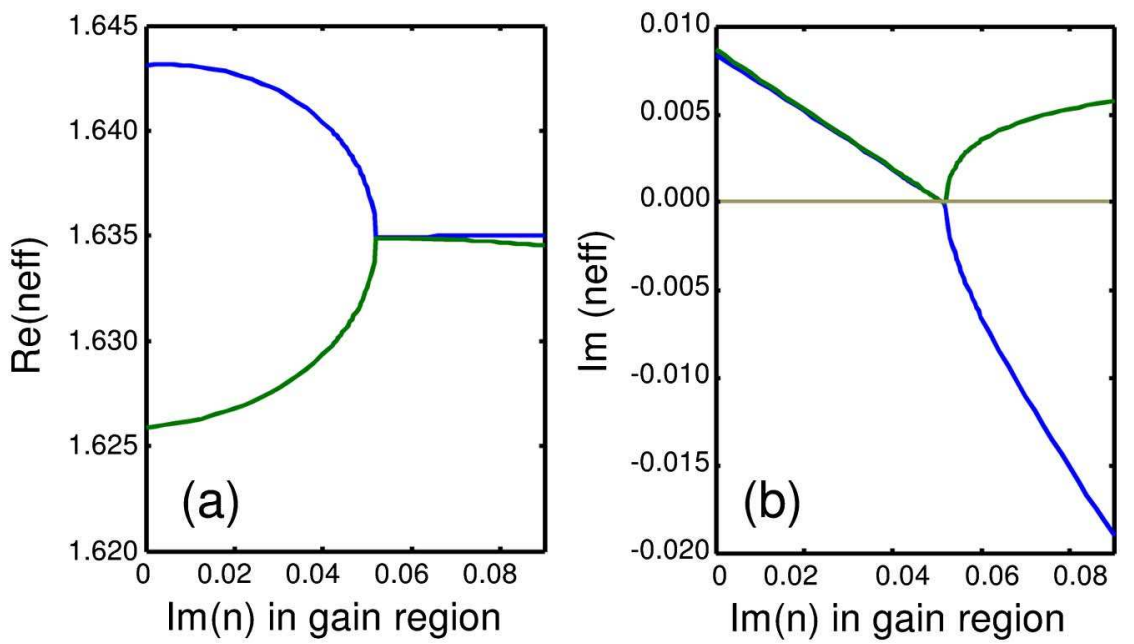

Fig. 10. (Color online) Exceptional point restoration: by adding complex gain (i.e., simultaneous gain and index change) in the region above the inverse-rib [see Fig. 9(a)], a nearly perfect singularity in the vicinity of the exceptional point can be restored. (a) Real part of $n_{\text {eff }}$ and (b) imaginary part of $n_{\text {eff }}$. 
singularities of imperfect PT-symmetric systems (most of those based on plasmonic waveguides, by the way), is general; we will publish its details elsewhere. The present example clearly suggests that even relatively extreme plasmonic structures can benefit from the special trends around a sharp singularity on nearly the same footing as had been demonstrated for less extreme ones such as LRSPP [22].

The difficulty of imposing a particular change of both the real and imaginary index in a prescribed and abruptly bounded region can, in practice, be replaced by two feasible tasks: (i) obtainment of a proper knowledge of all the electrooptical properties as a function of the local pumping intensity on a model system and (ii) choice of a smooth distribution of excitation intensity (optical pumping) across the waveguide section, as can be provided by few optogeometric parameters (central beam position, peak wavelength, and use of local absorption and field concentration/cancellation effects).

\section{CONCLUSIONS}

In this study, we have addressed issues that arose from our PIROW proposal [5], a hybrid waveguide with limited losses that can, in principle, be elaborated with simple technology, including a number of basic functions. The first part of the study has clarified that the optimum PIROW angle is around $35^{\circ}-50^{\circ}$, which can be understood as an essentially electrostatic effect: a sharper angle does not bring near the metal enough high-index material to shrink the mode, thus a rather deconfined mode is formed; conversely, a larger angle delocalizes the mode laterally.

We have addressed the issue of losses and shown that it had only a weak dependence on the tip angle. Hence the best compromise between mode area and losses should typically lay at the bottom of the $35^{\circ}-50^{\circ}$ range, around $35^{\circ}-40^{\circ}$. The spectral behavior of gold and silver PIROWs has also been examined, with trends being in agreement with similar hybrid waveguide approaches.

Next, we have examined the capability of a PIROW to channel SpE specifically from vertical dipoles. The trends on Purcell factor are dominated by the coupling to the PIROW mode and thus essentially follow the mode profile. A large Purcell factor up to $F_{p} \sim 8$ can be obtained for a vertical dipole lying close to the low-index gap in the high-index material and a factor $>30$ in the gap itself. In the latter case, a large fraction of emission goes to the metal. For a typical good compromise, the amount of light collected in the first micrometer can be as high as $85 \%$ on both sides.

Finally, we have capitalized on our recent approach [22] to fully consider the benefit of mimicking perfect PT-symmetric systems with two coupled waveguides, one with the fixed plasmonic losses and the other having added optical gain in and around the inverse-rib region. Without precautions, the singularity appears to be dangerously smoothed, which means that the capability to transform a small gain variation into a large difference in the coupled waveguide transfer function could seem jeopardized. However, a singularity "healing" mechanism was proposed, whereby pumping is assumed to induce a complex index change, typically $i g(1-0.174 i)$ instead of the pure imaginary contribution ig in our case. It could restore the singularity nearly entirely. Work is in progress to provide a more general basis to this mechanism.

\section{ACKNOWLEDGMENTS}

We thank Yan Chen for useful discussions on PT-symmetric systems during his master's first year internship.

\section{REFERENCES}

1. R. F. Oulton, V. J. Sorger, T. Zentgraf, R.-M. Ma, C. Gladden, L. Dai, G. Bartal, and X. Zhang, "Plasmon lasers at deep subwavelength scale," Nature 461, 629-632 (2009).

2. M. A. Noginov, G. Zhul, A. M. Belgrave, R. Bakker, V. M. Shalaev, E. E. Narimanov, S. Stout, E. Herz, T. Suteewong, and U. Wiesner, "Demonstration of a spaser-based nanolaser," Nature 460, 1110-1112 (2009).

3. R. F. Oulton, V. J. Sorger, D. A. Genov, D. F. P. Pile, and X. Zhang, "A hybrid plasmonic waveguide for subwavelength confinement and long-range propagation," Nat. Photon. 2, 496-500 (2008).

4. J. A. Dionne, L. A. Sweatlock, M. T. Sheldon, A. P. Alivisatos, and H. A. Atwater, "Silicon-based plasmonics for on-chip photonics," IEEE J. Sel. Top. Quantum Electron. 16, 295-306 (2010).

5. H. Benisty and M. Besbes, "Plasmonic inverse rib waveguiding for tight confinement and smooth interface definition," J. Appl. Phys. 108, 063108 (2010).

6. D. X. Dai and S. L. He, "A silicon-based hybrid plasmonic waveguide with a metal cap for a nano-scale light confinement," Opt Express 17, 16646-16653 (2009).

7. M. Fujii, J. Leuthold, and W. Freude, "Dispersion relation and loss of subwavelength confined mode of metal-dielectric-gap optical waveguides," IEEE Photon. Technol. Lett. 21, 362-364 (2009).

8. X. Y. Zhang, A. Hu, J. Z. Wen, T. Zhang, X. J. Xue, Y. Zhou, and W. W. Duley, "Numerical analysis of deep sub-wavelength integrated plasmonic devices based on semiconductor-insulatormetal strip waveguides," Opt. Express 18, 18945-18959 (2010).

9. A. V. Krasavin and A. V. Zayats, "Numerical analysis of longrange surface plasmon polariton modes in nanoscale plasmonic waveguides," Opt. Lett. 35, 2118-2120 (2010).

10. J. Ctyroky, V. Kuzmiak, and S. Eyderman, "Waveguide structures with antisymmetric gain/loss profile," Opt. Express 18, 21585-21593 (2010).

11. M. Kulishov, J. M. Laniel, N. Bélanger, and D. V. Plant, "Trapping light in a ring resonator using a grating-assisted coupler with asymmetric transmission," Opt. Express 13, 3567-3578 (2005).

12. S. Klaiman and L. S. Cederbaum, "Non-Hermitian Hamiltonians with space-time symmetry," Phys. Rev. A 78, 062113 (2008).

13. S. Klainman, U. Günther, and N. Moiseyev, "Visualization of branch points in PT-symmetric waveguides," Phys. Rev. Lett. 101, 080402 (2008).

14. K. G. Makris, R. El-Gaininy, D. N. Christodoulides, and Z. H Musslimani, "Beam dynamics in PT symmetric optical lattices," Phys. Rev. Lett. 100, 103904 (2008).

15. O. Bendix, R. Fleischmann, T. Kottos, and B. Shapior, "Exponentially fragile PT symmetry in lattices with localized eigenmodes," Phys. Rev. Lett. 103, 030402 (2009).

16. J. J. Chen, Z. Li, S. Yue, and Q. H. Gong, "Hybrid long-range surface plasmon-polariton modes with tight field confinement guided by asymmetrical waveguides," Opt. Express 17, 2360323609 (2009).

17. A. Guo, G. J. Salamo, R. Duchesne, R. Morandotti, M. VolatierRavat, V. Aimez, G. A. Siviloglou, and D. N. Christodoulides, "Observation of PT-symmetry breaking in complex optical potentials," Phys. Rev. Lett. 103, 093902 (2009).

18. T. Kottos, "Broken symmetry makes light work," Nat. Phys. 6 166-167 (2010).

19. C. E. Rüter, K. G. Makris, R. El-Gaininy, D. N. Christodoulides, M. Segev, and D. Kip, "Observation of parity-time symmetry in optics," Nat. Phys. 6, 192-195 (2010).

20. A. A. Sukhorukov, Z. Xu, and Y. Kivshar, "Nonlinear breaking of PT symmetry in coupled waveguides with balanced gain and loss," in Nonlinear Photonics, OSA Technical Digest (CD) (Optical Society of America, 2010), paper NTuC19.

21. C. T. West, T. Kottos, and T. Prosen, "PT-symmetric wave chaos," Phys. Rev. Lett. 104, 054102 (2010).

22. H. Benisty, A. Degiron, A. Lupu, A. De Lustrac, S. Chenais, S Forget, M. Besbes, G. Barbillon, A. Bruyant, S. Blaize, and G. 
Lerondel, "Implementation of PT symmetric devices using plasmonics: principle and applications," Opt. Express 19, 18004-18019 (2011).

23. A. Degiron, S. Y. Cho, T. Tyler, N. M. Jokerst, and D. R. Smith, "Directional coupling between dielectric and long-range plasmon waveguides," New J. Phys. 11, 015002 (2009).

24. P. B. Johnson and R. W. Christy, "Optical constants of the noble metals," Phys. Rev. B 6, 4370-4379 (1972).

25. J.-M. Jin, The Finite Element Method in Electromagnetics, 2nd ed. (Wiley-IEEE, 2002).

26. R. F. Oulton, G. Bartal, D. F. P. Pile, and X. Zhang, "Confinement and propagation characteristics of subwavelength plasmonic modes," New J. Phys. 10, 105018 (2008).

27. J. Ctyroky, S. Helfert, R. Pregla, P. Bienstman, R. Baets, R. De Ridder, R. Stoffer, G. Klaasse, J. Petracek, P. Lalanne,
J. P. Hugonin, and R. M. De La Rue, "Bragg waveguide grating as a 1D photonic band gap structure: COST 268 modelling task," Opt. Quantum Electron. 34, 455-470 (2002).

28. W. Lukosz and R. E. Kunz, "Fluorescence lifetime of magnetic and electric dipoles near a dielectric interface," Opt. Commun. 20, 195-199 (1977).

29. R. Esteban, T. V. Teperik, and J. J. Greffet, "Optical patch antennas for single photon emission using surface plasmon resonances," Phys. Rev. Lett. 104, 026802 (2010).

30. G. W. Ford and W. H. Weber, "Electromagnetic interaction of molecules with metal surfaces," Phys. Rep. 113, 195-287 (1984).

31. Z. Han, A. Y. Elezzabi, and V. Van, "Experimental realization of subwavelength plasmonic slot waveguides on a silicon platform," Opt. Lett. 35, 502-504 (2010). 